\title{
Analysis of Dynamic Atrial Dimension and Function during Early Cardiac Development in the Chick Embryo
}

\author{
KATHLEEN A. CAMPBELL, NORMAN HU, EDWARD B. CLARK, AND BRADLEY B. KELLER \\ Strong Children's Research Center, Dorothy S. and Frederick W. Cook Research Laboratory, Division of \\ Pediatric Cardiology, University of Rochester School of Medicine, Rochester, New York 14642
}

\begin{abstract}
Although atrial morphologic changes are well documented, the description of early atrial function is limited. We used videomicroscopic methods to define the function of the contracting atrium in stage 16 to 24 white Leghorn chick embryos. We exposed the embryo in ovo (right side up) and imaged the ventricle, then repositioned the embryo (left side up) and imaged the atrium ( $n \geq 8$ per stage). We traced the atrial endocardial border and then measured atrial perimeter $(\mathrm{mm})$ and cross-sectional area $\left(\mathrm{mm}^{2}\right)$. A 20-MHz pulsed Doppler velocity meter was used to measure atrioventricular blood velocity during atrial imaging in an additional six stage 21 embryos. Data were tested by analysis of variance and regression analysis. Mean heart rate change after repositioning was $-4 \pm 1 \%$. Atrial maximum and minimum area increased linearly versus embryo stage $(y=0.10 x-1.41, r=0.89, p<0.05$ and $y=0.05 x-0.67, r=0.82, p<0.05$, respectively). Shortening fraction (percentage of reduction) of atrial perimeter and area decreased from $32.3 \pm 2.0 \%$ to $27.5 \pm$ $1.8 \%(p<0.05)$ and $56.2 \pm 3.0 \%$ to $47.7 \pm 2.0 \%(p<$ 0.05 ), respectively, from stage 16 to 24 . During atrial contraction, the velocity of circumferential wall shortening increased linearly with stage $(y=0.22 x-2.08, r=0.81$, $p<0.01$; however, the velocity of lengthening was similar between stages $(p=0.45)$. Simultaneous atrial imaging and pulsed Doppler velocity measurement showed that passive atrioventricular flow occurred late in atrial lengthening and active atrioventricular flow occurred during atrial contraction. Thus, atrial function increases in parallel with morphogenesis during early cardiac development, and measures of atrial function can now be incorporated into a physiologic model of the developing cardiovascular system. (Pediatr Res 32: 333-337, 1992)
\end{abstract}

Abbreviations

$\mathrm{AV}$, atrioventricular

The chick embryo has been used to explore fundamental mechanisms of cardiovascular development for over a century (1). Like the mammalian heart, the avian heart develops from a

Received February 10, 1992; accepted April 28, 1992.

Correspondence and reprint requests: Bradley B. Keller, M.D., University of Rochester, Strong Children's Research Center, Division of Pediatric Cardiology, 601 Elmwood Ave., Box 631, Rochester, NY 14642.

Supported by NIH NRSA HL07824 (B.B.K.), NIH PSA HL02498 (B.B.K.) NIH RO1 HL42151 (E.B.C.), Donald L. Shapiro Student Research Award (K.A.C.) and University of Rochester Medical Student Research Fellowship (K.A.C.).

'Presented in part at the Annual Meeting of the Society for Pediatric Research/ American Pediatric Society, New Orleans, LA, May 1991. smooth-walled, pulsatile tube into a four-chambered pump while simultaneously meeting the metabolic demands of the rapidly growing embryo (2). Growth and function of the embryonic heart are related, and both can be experimentally altered during primary cardiac morphogenesis (3). In the chick, the primitive atrium is formed by the fusion of the paired primordia at stage 9 (h 29-33 of a $21-\mathrm{d}$ incubation period) (4). The atrium functions as a single chamber until stage $29(6 \mathrm{~d})$, when atrial septation and fusion of the ventral and dorsal endocardial cushions are complete (5). In contrast to the trabeculated endocardium of the developing ventricle, the endocardial surface of the atrium is a smooth, nontrabeculated cell surface (6).

In the mature heart, atrial function has been described in terms of reservoir, conduit, and pump function (7). Atrial contractility, compliance, and contribution to ventricular filling have been studied using in vitro, animal, and computer models (8-11). To date, the size and location of the embryonic atrium has limited the definition of atrial function (12). How the atrium contributes to embryonic cardiovascular function has not been fully evaluated. Thus, to expand our understanding of embryonic ventricular function $(2,13-18)$ we defined changes in atrial function during primary cardiac morphogenesis.

In this study, we used videomicroscopic and pulsed Doppler techniques to describe atrial dimension and function during early cardiac development. We found that atrial contraction time and shortening fraction decreased with stage. Rate-corrected peak velocity of wall contraction increased with stage, whereas ratecorrected peak velocity of lengthening remained similar. Passive and active AV blood flow, as defined by pulsed Doppler velocity meter, correlated with atrial lengthening and contraction. These measures of atrial function can now be incorporated into a physiologic model of the developing cardiovascular system.

\section{MATERIALS AND METHODS}

We studied white Leghorn chick embryos at stage 16 ( $2.3 \mathrm{~d}, n$ $=8)$, stage $18(2.8 \mathrm{~d}, n=11)$, stage 21 ( $3.5 \mathrm{~d}, n=14)$, and stage $24(4 \mathrm{~d}, n=10)$ of a 46 -stage $(21-\mathrm{d})$ incubation period. Each stage was identified by visual landmarks that include somite number, limb size, and cardiac morphology (19). Embryo wet weight doubles between each selected stage (2). Fertile eggs were incubated blunt end up in a forced draft incubator at $38.5^{\circ} \mathrm{C}$. Access to the embryo was gained by opening the shell, removing a small region of extraembryonic membranes, then positioning the embryo for imaging on a stereophotomacroscope stage. Embryo temperature was maintained by two ambient warmers.

Video images were acquired using a stereophotomacroscope (model M400 Wild, Wild Leitz USA, Inc., Rockleigh, NJ), a Dage 70 series video camera (Dage-MTI, Michigan City, IN) with a grade 1 Newvicon tube, a fiber optic light source (DolanJenner Industries, Woburn, MA), a Magnavox PV9670 VHS video recorder, and a time-date generator (FOR.A model VTG- 
33, FOR.A, West Newton, MA) (Fig. 1). The video camera generated 60 sequential video fields per $s$ in the interlaced mode. The image field was $1000-2000 \mu \mathrm{m}$ in diameter, and the effective raster spacing was $4-8 \mu \mathrm{m}$. Real time $\pm 0.005 \mathrm{~s}$ was recorded on each field. A $100-\mu$ m division scribed glass standard was recorded in the focal plane of each embryo after imaging.

Individual video fields were analyzed at a work station that included a minicomputer (Premium/286, AST Research, Irvine, CA), a monitor (Multisync II, NEC Information Systems, Boxborough, MA), a frame grabbing board (model M8 TARGA, Jandel Scientific, Corte Madera, CA), JAVA video analysis software (Jandel Scientific), a Microsoft mouse, and a multipurpose video monitor (Sony PVM1271Q). AV blood flow was measured using a $20-\mathrm{MHz}$ pulsed Doppler velocity meter (University of Iowa, Dept. of Bioengineering). Because of the dynamic diameter of the AV orifice, blood flow velocity was used only to identify the onset of passive and active ventricular filling. This Doppler velocity meter has been validated to be accurate up to $16 \mathrm{~mm} / \mathrm{s}$ (2).

Analog wave forms were sampled at $500 \mathrm{~Hz}$ by a Computerscope data acquisition analog/digital board (RC Electronics, Inc., Santa Barbara, CA) and displayed on the computer screen in digital oscilloscope mode. Additionally, the analog Doppler wave form was superimposed on the video images in real time using a custom analog device (PONV, Ogden Scientific, Spencerport, NY). Simultaneous measurement of atrial dimension and AV blood flow velocity enabled identification of the onset of atrial contraction and lengthening.

Experimental protocol. Each embryo was imaged for $60 \mathrm{~s}$ in normal right-side up position with the ventricle exposed. The embryo was then gently turned left side up, exposing the atrium (Fig. 2), and imaging continued for $5 \mathrm{~min}$. We recorded the image of a glass scribed standard at the end of each experiment for use in calibration of the video measurement software. The

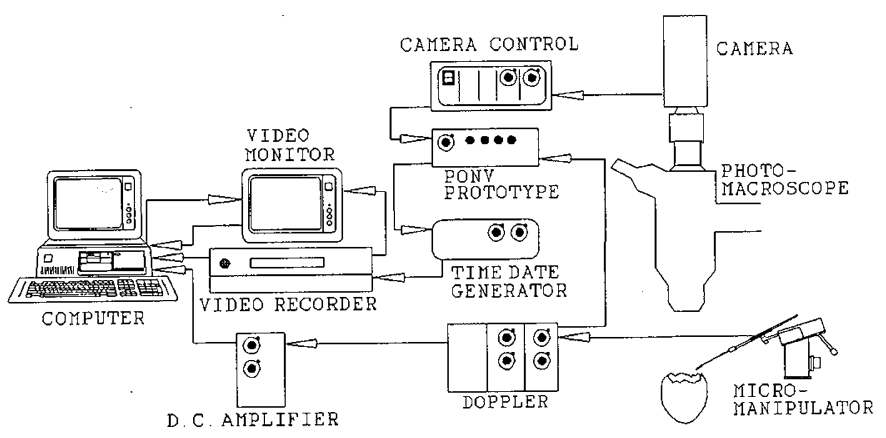

Fig. 1. Diagram of videomicroscopy work station.

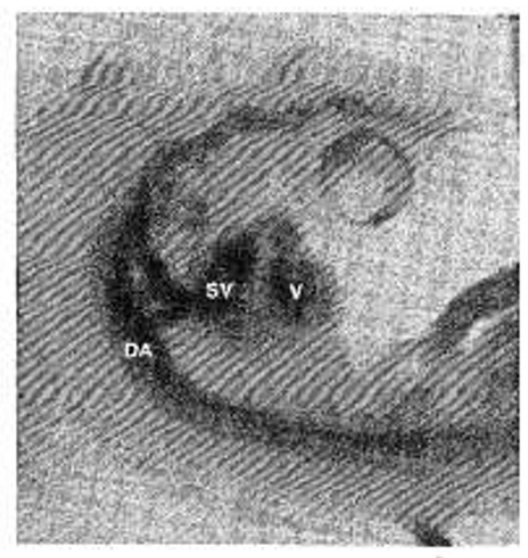

RIGHT SIDE UP video recordings of each embryo were reviewed in slow motion to follow the pattern of blood flow from the venous bed to the sinus venosus and from the atrium to the ventricle. At this speed, we identified individual red blood cells and followed their direction of flow. We traced atrial endocardial perimeter $(\mathrm{mm})$ and cross-sectional area $\left(\mathrm{mm}^{2}\right)$ in sequential video fields (Fig. 3). In a subset of stage 21 embryos $(n=6)$, we simultaneously measured AV blood velocity and recorded video images. At this stage, the AV canal is undivided and demarcated by the anterior and posterior endocardial cushions (5). With the embryo left side up, a $0.75-\mathrm{mm}$ piezoelectric crystal was positioned on the upper epicardial surface of the atrium, parallel to the AV orifice. The angle of inclination and Doppler meter range were varied to maximize the amplitude of the AV velocity profile.

Data analysis protocol for each embryo. For each embryo, 1) perimeter and area data were smoothed with a 3-point Fast Fourier Transform low-pass filter; 2) heart rate (bpm) and cycle length (ms) were calculated from the time record of consecutive maximum area images; 3) atrial contraction time (ms) was calculated as the time from onset of contraction to onset of lengthening and indexed by dividing contraction duration by the cycle length; 4) maximum and minimum atrial perimeter (mm) and area $\left(\mathrm{mm}^{2}\right)$ were determined from three cardiac cycles; 5) shortening fraction $(\mathrm{SF})$ was calculated for perimeter $(\mathrm{P})$ and area $(\mathrm{A}): \mathrm{SF}=[($ maximum - minimum $) /$ maximum $] \times 100 ; 6)$ peak velocity of circumferential wall shortening and lengthening was calculated from perimeter $(\mathrm{mm} / \mathrm{s})$; and 7$)$ time course of passive and active AV blood flow was compared to simultaneous change in atrial perimeter.

Statistical analysis. Data are reported as mean \pm SEM, $n \geq 8$ for each stage. The mean values for each variable were compared by analysis of variance and regression analysis across the stage range, and $p<0.05$ was assigned significance. Intra- and interobserver errors of planimetry of atrial endocardial perimeter on 10 pairs of distinct images were not significant (mean $=0.55$ $\mathrm{mm}^{2}, \mathrm{SEM}=0.03 \mathrm{~mm}^{2}, p=0.68$ and mean $=0.53 \mathrm{~mm}^{2}, \mathrm{SEM}$ $=0.03 \mathrm{~mm}^{2}, p=0.98$, respectively).

\section{RESULTS}

The atrium was visualized in most embryos after repositioning. Venous return from the extra-embryonic circulation was nonpulsatile. A small amount of pulsatile retrograde blood flow was seen moving from the sinus venosus to the cardinal veins. At each stage, the embryonic atrium had both a rapid and slow filling phase and a contraction phase (Fig. 4).

The mean change in heart rate after embryo repositioning from right to left side up was $-4 \pm 1 \%$ (Fig. 5). Atrial contraction time decreased from $140 \pm 5$ to $92 \pm 3 \mathrm{~ms}$ from stage 16 to 24

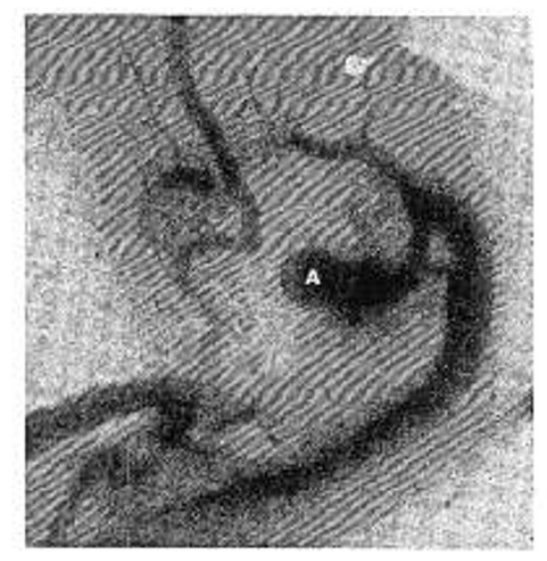

\footnotetext{
Fig. 2. Stage 18 chick embryo, right side up and left side up. $D A$, dorsal aorta; $S V$, sinus venosus; $V$, ventricle; $A$, atrium.
} 


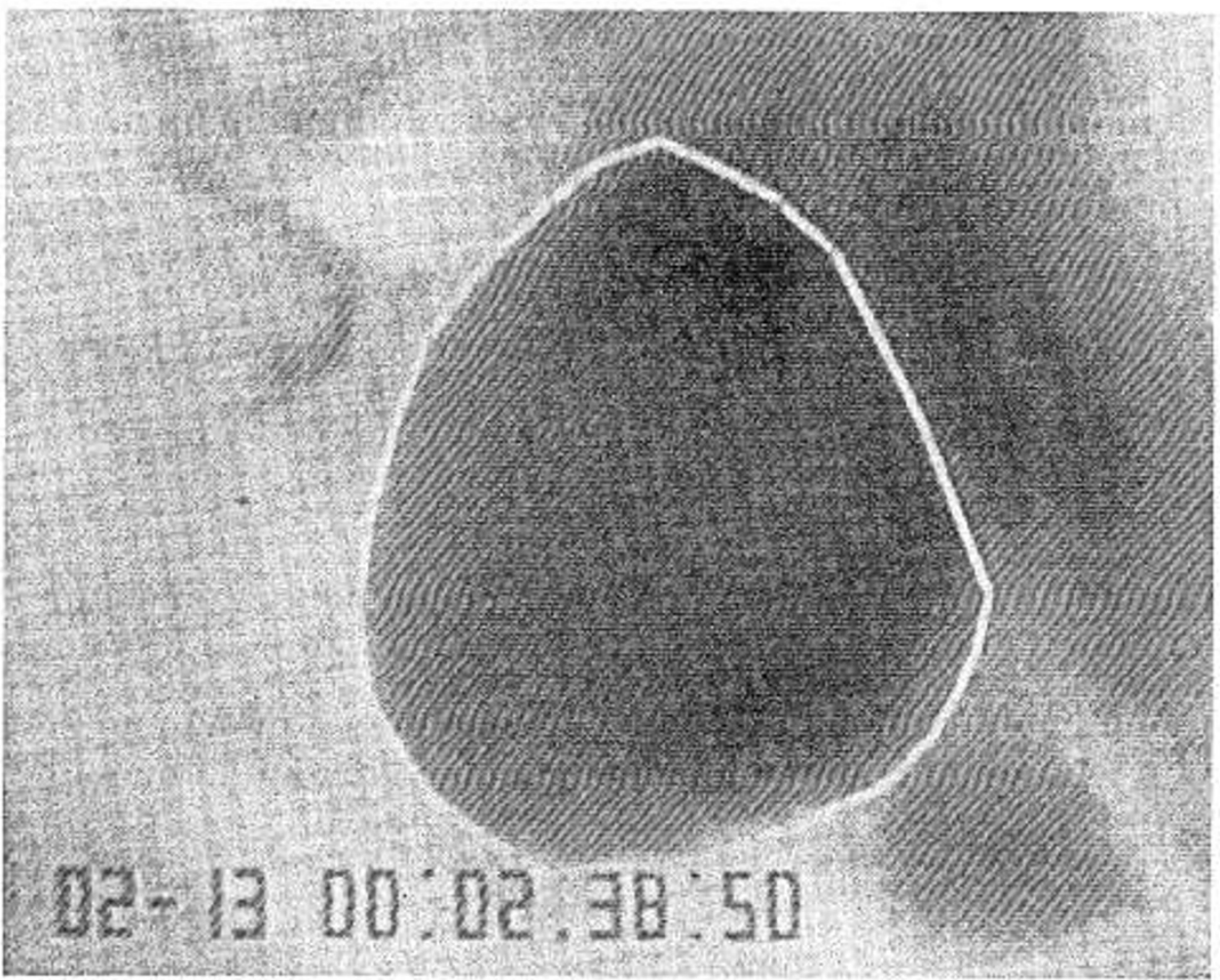

Fig. 3. Embryonic atrium planimetered for perimeter and area.

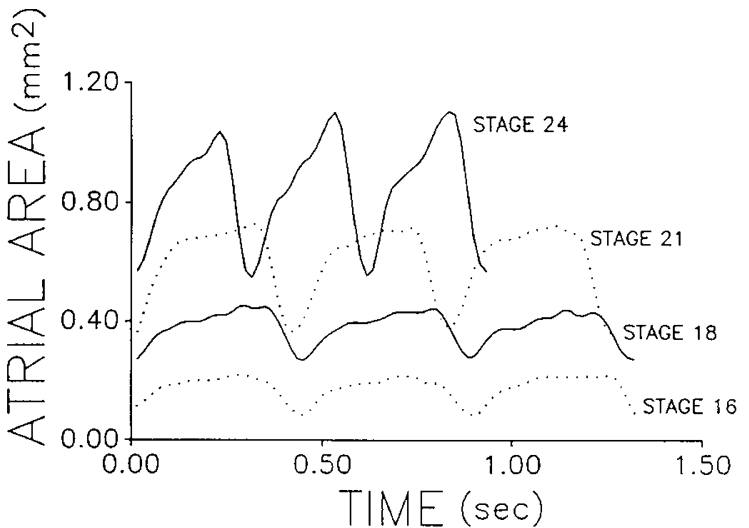

Fig. 4. Time record of dynamic atrial area. Curves plotted on the same time scale. Data displayed as line drawings without curve fitting.

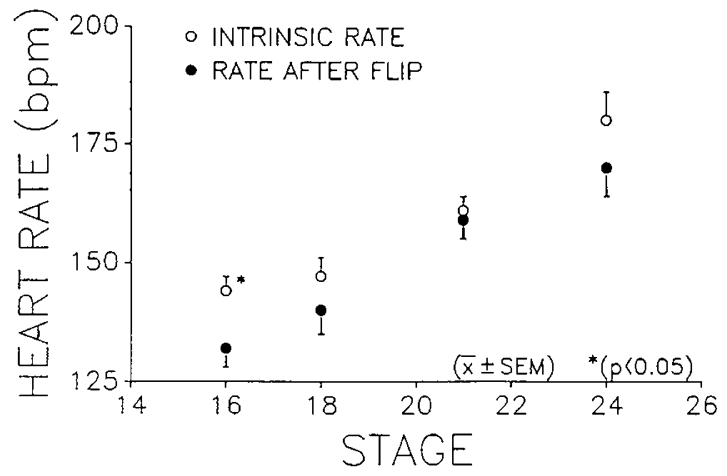

Fig. 5. Heart rate before and after flip. Data plotted are mean \pm SEM.

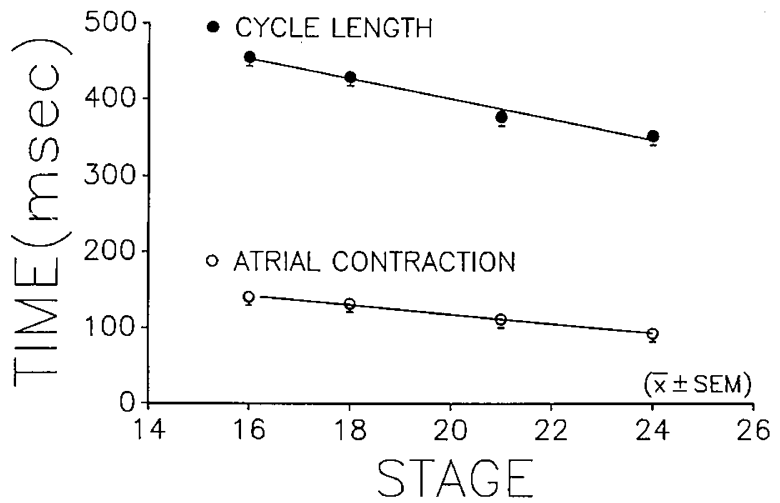

Fig. 6. Cycle length and corrected atrial contraction time. Data plotted are mean \pm SEM. Linear regression curves plotted to raw data.

$(p<0.01$, Fig. 6). The duration of atrial contraction indexed for cycle length decreased from $30.8 \pm 2.4 \%$ to $26.1 \pm 2.4 \%$ from stage 16 to $24(p<0.05)$.

Simultaneous pulsed Doppler and atrial imaging showed that passive AV flow occurred late in atrial lengthening and active AV flow occurred during atrial contraction (Fig. 7). Anterograde blood flow through the AV cushions occurred during ventricular diastole, and no retrograde flow was measured during ventricular systole.

Maximum and minimum atrial perimeter increased linearly versus stage $(y=0.25 x-2.17, r=0.91, p<0.05$ and $y=0.19 x$ $-1.82, r=0.91, p<0.05$, respectively, Fig. 8). Maximum and minimum atrial area also increased linearly versus embryo stage $(y=0.01 x-1.41, r=0.89, p<0.05$ and $y=0.05 x-0.67, r=$ $0.82, p<0.05$, respectively, Fig. 9 ).

Shortening fractions for atrial perimeter decreased from 32.3 $\pm 2.0 \%$ to $27.5 \pm 1.8 \%$ and for area from $56.2 \pm 3.0 \%$ to 47.7 $\pm 2.0 \%$ from stage 16 to 24 ( $p<0.05$ for each). Peak velocity of circumferential wall shortening occurred shortly after the onset of atrial contraction and increased linearly with stage $(y=0.22 x$ 


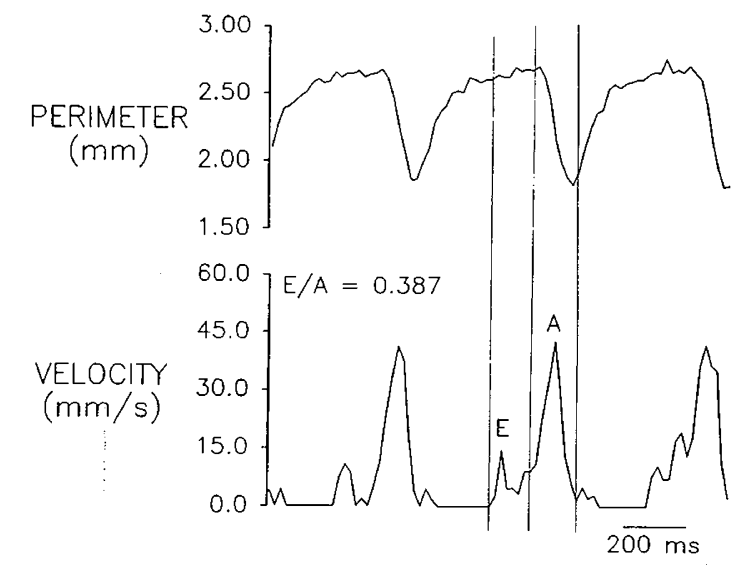

Fig. 7. Simultaneous dynamic atrial perimeter and AV blood velocity. $E$ represents passive flow and $A$ represents active flow.

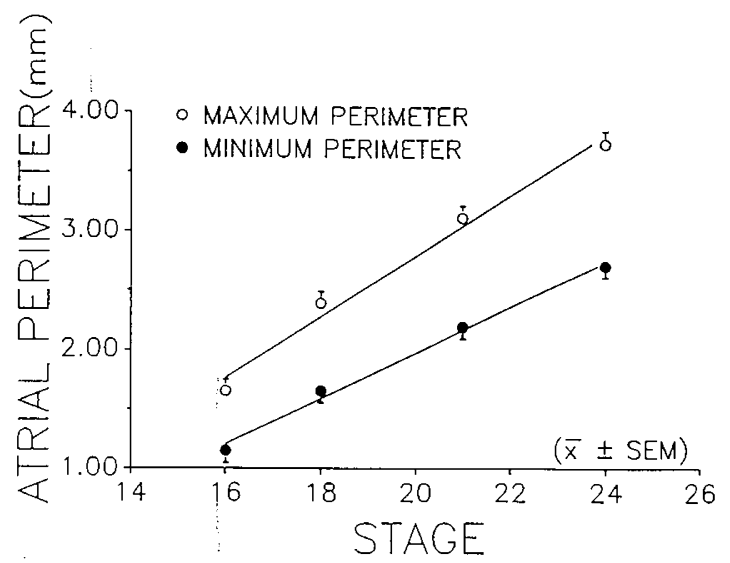

Fig. 8. Atrial maximum and minimum perimeter. Data plotted are mean \pm SEM

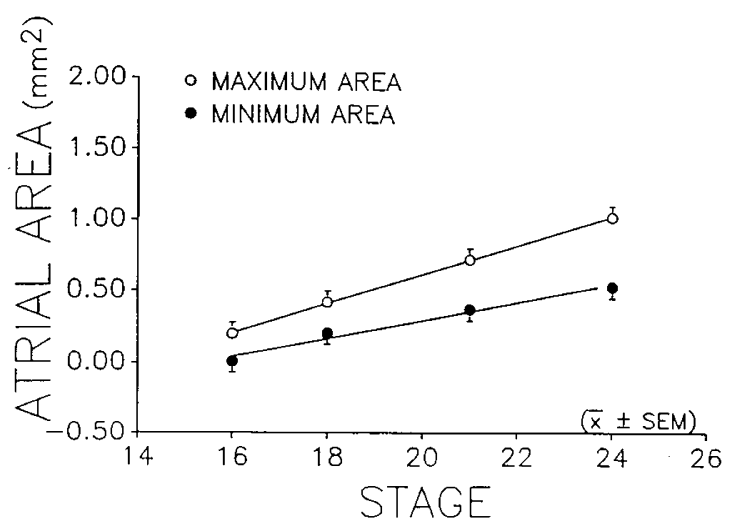

Fig. 9. Atrial maximum and minimum area. Data plotted are mean SEM.

$-2.08, r=0.81, p<0.01)$. Peak velocity of lengthening occurred immediately after the completion of atrial contraction and was similar between stages 18 and 24 ( $p=0.45$, Fig. 10).

\section{DISCUSSION}

The accurate measurement of atrial function is critical to understand the mechanical properties of the atrium and to define the effect of atrial function on ventricular and vascular development. Our study combined pulsed Doppler measures of AV blood velocity and videomicroscopic measures of atrial size to define atrial function during primary cardiac morphogenesis.

Although the sinoatrial junction was not well visualized, trivial retrograde flow was visualized from the sinus venosus to the

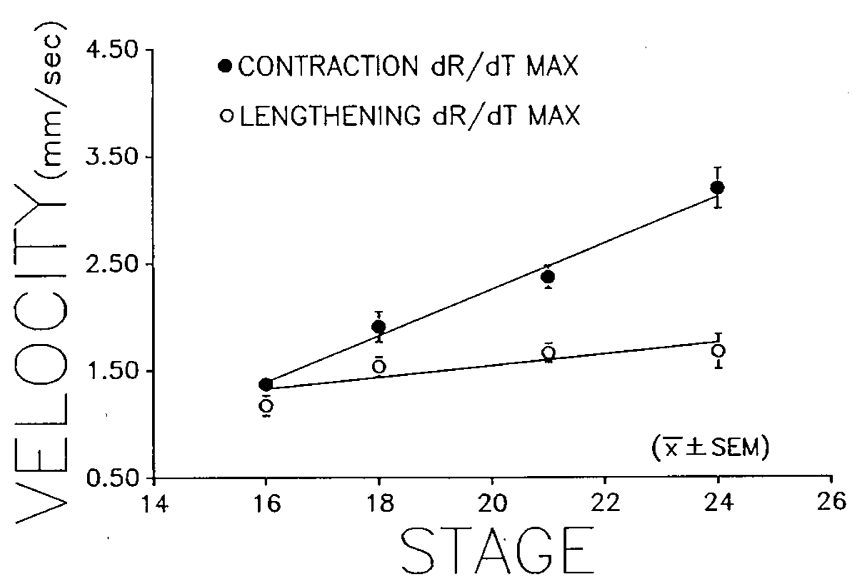

Fig. 10. Peak atrial contraction and lengthening velocities. Data plotted are mean \pm SEM.

cardinal veins. Pulsed Doppler interrogation of the human sinoatrial junction during the 2 nd and 3 rd trimesters of gestation revealed a small degree of retrograde flow during atrial contraction (16). This separation of low pressure venous return from atrial contraction likely depends on the dynamic collapse or constriction of the venoatrial junction. All embryonic AV flow was anterograde, consistent with previous studies that demonstrate that the AV cushions act as valves to prevent retrograde flow during ventricular systole $(17,18)$.

Heart rate increases during cardiac morphogenesis (2). In contrast, cardiac intervals, such as ventricular ejection time, remain similar when indexed for cycle length (20). We found that despite a $34 \%$ decrease in atrial contraction time the duration of atrial contraction indexed for cycle length ranged from $30.8 \pm 2.4 \%$ to $26.1 \pm 2.4 \%$ from stage 16 to 24 . The ability of the developing heart to fill and eject at progressively faster rates likely reflects the simultaneous maturation of organ geometry, tissue architecture, and cellular pathways for force generation. Supporting this maturational progression are various experiments designed to perturb embryonic heart rate that consistently show that peak performance occurs at the intrinsic rate $(14,21-$ 23).

Evaluation of simultaneous dynamic atrial perimeter and AV blood velocity confirmed that atrial contraction duration corresponds to the time of active AV blood flow. Unfortunately, video imaging could not quantify passive AV flow because there was no corresponding change in atrial perimeter. Similarly, pulsed Doppler velocity measurement cannot directly measure passive AV flow because AV orifice diameter is dynamic.

Atrial shortening fractions were within a narrow range from stage 16 to 24 despite a 16-fold increase in ventricular mass. This is consistent with basic sarcomere mechanics favoring an optimal resting length (24). Dynamic two-dimensional changes in surface area, termed strains, reflect sarcomere length changes on a tissue level. These data on atrial contraction are consistent with the $20 \%$ peak epicardial strains measured in the stage 16 chick ventricle (25) and with epicardial strain measurements of the mature myocardium (26). This finding is remarkable because the early embryonic myocyte lacks myofibrillar alignment (2). A complete analysis of atrial epicardial strains is needed to better define regional atrial contraction and material properties of the developing atrium.

There are limitations to videomicroscopic techniques. Video imaging alone cannot accurately quantify the amount of blood stored in the atrium during ventricular systole (reservoir function) or the flow of blood from sinus venosus to ventricle during ventricular filling (conduit function). Simultaneous AV blood velocity, atrial pressure, and video imaging during alterations in atrial preload are necessary to define atrial and ventricular pressure and flow relationships. 
We noted a marked increase in rate-corrected atrial circumferential shortening velocity with development. The contraction velocity of mature ventricular myocardium is directly related to preload and contractile state and inversely related to afterload (27). Preload recruitment is probably not responsible for the increased contraction velocity because shortening fraction actually decreased slightly from stage 16 to 24 . Maturational changes in atrial myosin heavy chains from a mixture of ventricular and atrial isoforms to exclusively atrial isoforms would increase contraction velocity (28). Further experiments are needed to correlate the peak velocity of atrial circumferential wall shortening to atrial preload, contractile state, and ventricular compliance.

In contrast, peak velocity of lengthening was similar between stages after stage 16 . In the mature myocardium, peak velocity of relaxation is related to the uptake of calcium by the sarcoplasmic reticulum and to myocardial viscoelastic properties (29). Calcium flux in embryonic myocytes occurs primarily across the sarcolemma because of the lack of an organized sarcoplasmic reticulum or t-tubule system in myocytes at these stages (30). Unfortunately, the viscoelastic properties of the embryonic myocardium have not been defined. Thus, further information on embryonic calcium transport and myocardial viscoelastic properties are needed to better understand myocardial relaxation during cardiac development.

The time course and magnitude of AV blood flow depends upon a dynamic interaction between the atrium, AV cushion, and ventricle. The atrium increases dramatically in size and function from stage 16 to 24 . Dramatic remodeling of the ventricular wall occurs coincident with atrial maturation. Thus, at each embryonic stage a unique combination of atrial and ventricular parameters of pressure, flow, and compliance are present. Experiments in the chick embryo designed to chronically alter AV blood flow support the hypothesis that ventricular development depends upon adequate AV flow (31). Further experiments are needed to define simultaneous atrial and ventricular pressureflow relationships during normal and altered AV blood flow.

The definition of normal atrial function in the chick embryo broadens our foundation for investigating the interrelationship of atrial and ventricular function during cardiovascular development. The combined analysis of atrial and ventricular function using videomicroscopic, pulsed Doppler, and servo-null pressure techniques expands the utility of the chick embryo as a model of normal and experimentally altered cardiovascular development.

Acknowledgment. The authors thank Joseph P. Tinney for his skillful technical assistance.

\section{REFERENCES}

1. Pickering JW 1893 Observations on the physiology of the embryonic heart. J Physiol (Lond) 14:383-466

2. Hu N, Clark EB 1989 Hemodynamics of the stage 12 to stage 29 chick embryo. Circ Res 65:1665-1670

3. Clark EB, Hu N, Frommelt P, Dumett JL, Tomanek RJ 1989 Effect of increased pressure on ventricular growth in stage 21 chick embryo. Am J Physiol 257:H55-H61

4. Manasek FJ 1968 Embryonic development of the heart: I. A light and electron microscopic study of myocardial development in the early chick embryo. J Morphol 125:329-336

5. Hay DA, Low FN 1970 The fusion of dorsal and ventral endocardial cushions in the embryonic chick heart: a study in fine structure. Am J Anat 133:124

6. Patten BM 1971 The circulatory system. In: Patten BM (ed) Early Embryology of the Chick. McGraw-Hill, New York, pp 202-223

7. Nikolic SD, Biascucci LM, Tanoue T, Liao KX, Solomon S, Jondeau G, Nanna M, Frater RW, Yellin EL 1990 Separation of the left atrial reservoir, conduit and pump functions by controlled atrial filling. Circ 82(Suppl III):III606(abstr)

8. Blinks JR, Koch-Weser J 1961 Analysis of the effects of changes in rate and rhythm upon myocardial contractility. J Pharmacol Exp Therap 134:373389

9. Williams JF, Sonnenblick EH, Braunwald E 1965 Determinants of atrial contractile force in the intact heart. Am J Physiol 209:H1061-H1068

10. Suga H 1974 Importance of atrial compliance in cardiac performance. Circ Res 35:39-43

11. Lau V, Sagawa K 1979 Model analysis of the contribution of atrial contraction to ventricular filling. Ann Biomed Eng 7:167-201

12. Sol P 1981 Development of blood pressure in the atria of the chick embryo and its relationship with the formation of the interatrial septum. MD thesis, University of Lausanne School of Medicine, Institute of Histology and Embryology, Lausanne, Switzerland

13. Faber JJ, Green TJ, Thornburg KL 1974 Embryonic stroke volume and cardiac output in the chick. Dev Biol 41:14-21

14. Zimmerman FJ, Hughes S, Cuneo B, Benson DW 1991 The effect of cycle length on ventricular end-diastolic pressure and maximum time derivative of pressure in the stage 24 chick embryo. Pediatr Res 29:338-341

15. Keller BB, Hu N, Clark EB 1990 Correlation of ventricular area, perimeter, and conotruncal diameter with ventricular mass and function in the stage 12 to 24 chick embryo. Circ Res 66:109-114

16. Reed KL, Appleton CP, Anderson CF, Shenker L, Sahn DJ 1990 Doppler studies of vena cava flows in human fetuses. Insights into normal and abnormal cardiac physiology. Circulation 81:498-505

17. Hu N, Connuck D, Keller BB, Clark EB 1991 Diastolic filling characteristics in the stage 12 to 27 chick embryo ventricle. Pediatr Res 29:334-337

18. Keller BB, Hu N, Serrino PJ, Clark EB 1991 Ventricular pressure-area loop characteristics in the stage 16 to 24 chick embryo. Circ Res 68:226-231

19. Hamburger V, Hamilton HL 1951 A series of normal stages in the development of the chick embryo. J Morphol 88:49-92

20. Hu N, Keller BB, Clark EB 1991 The effect of bradycardia on systolic and diastolic time intervals in the stage 16 to 27 chick embyro. Pediatr Res 29:19A(abstr)

21. Wispe JR, Hu N, Clark EB 1983 Effect of environmental hypothermia on dorsal aortic blood flow in the chick embryo, stages 18 to 24 . Pediatr Res 17:945-948

22. Nakazawa M, Miyagawa S, Takao A, Hu N, Clark EB 1986 Hemodynamic effects of environmental hyperthermia in the stage 18,21 , and 24 chick embryo. Pediatr Res 20:1213-1215

23. Dunnigan A, Hu N, Benson Jr DW, Clark EB 1987 Effect of heart rate increase on dorsal aortic blood flow in the stage 24 chick embryo. Pediatr Res 22:442444

24. ter Keurs HEDJ, Rijnsburger WH, van Heuningen R, Nagelsmit MJ 1980 Tension development and sarcomere length in rat cardiac trabeculae: evidence of length-dependent activation. Circ Res 46:703-714

25. Taber LA, Keller BB, Clark EB 1992 Experimental and theoretical ventricular strains in the stage 16 chick embryo. J Biomech Eng (in press)

26. Waldman LK, Fung YC, Covell JW 1985 Transmural myocardial deformation in the canine left ventricle: normal in vivo three-dimensional strains. Circ Res 57:152-163

27. Sonnenblick AH, Parmley WW, Urschel CW 1969 The contractile state of the heart as expressed by force-velocity relations. Am J Cardiol 23:488-503

28. Sweeney LJ, Zak R, Manasek FJ 1987 Transitions in cardiac isomyosin expression during differentiation of the embryonic chick heart. Circ Res 61:287-295

29. Barry WH 1988 Cellular mechanisms of relaxation: lessons from frogs, birds, and mammals. In: Grossman W, Lorell BH (eds) Diastolic Relaxation of the Heart. Martinus Nijhoff Publishing, Boston, pp 3-9

30. Nakanishi T, Seguchi M, Takao A 1988 Development of the myocardial contractile system. Experientia 44:936-944

31. Sweeney LJ, Clark EB, Rosenquist GC 1980 Left heart hypoplasia in the chick embryo: an experimental study. Anat Rec 196:186(abstr) 